\title{
HER2 breast cancer therapies: a review
}

\author{
This article was published in the following Dove Press journal: \\ Biologics: Targets \& Therapy \\ 9 June 2009 \\ Number of times this article has been viewed
}

\section{Conleth G Murphy Shanu Modi \\ Breast Cancer Medicine Service, Department of Medicine, Memorial Sloan-Kettering Cancer Center, New York, NY, USA}

\begin{abstract}
Amplification of the HER2 gene and/or overexpression of its protein product have been found in up to $25 \%$ to $30 \%$ of human breast cancers and have been shown to be associated with poorer outcomes compared to 'HER2 normal' breast cancer. Research has focused on developing therapies directed to the HER2 receptor and its pathway. These include the monoclonal antibody trastuzumab, which has improved outcomes when used in patients with both advanced and early breast cancer. Lapatinib is a small-molecule tyrosine kinase inhibitor which has demonstrated activity in advanced breast cancer and is currently being evaluated in early stage disease. We discuss the therapeutic rationale and clinical trial experience with these agents. Other novel and emerging strategies targeting the HER2 receptor and its pathway are also discussed. These strategies include novel HER2 antibodies and small-molecule inhibitors, antibody-drug conjugates, agents targeting downstream components of the HER2 signaling pathway, and heat shock protein 90 (HSP90) inhibitors.
\end{abstract}

Keywords: HER2, human epidermal growth factor receptor 2, breast cancer, trastuzumab, lapatinib

The human epidermal growth factor receptor 2 (HER2) gene, also known as c-erbB2 and $n e u$, encodes a $185-\mathrm{kDa}$ transmembrane glycoprotein receptor. This receptor belongs to the ErbB family of growth factor receptors with intrinsic tyrosine kinase activity, the members of which exist in homodimer and heterodimer form when activated. ${ }^{1}$ Other members of the ErbB family are the epidermal growth factor receptor 1 (EGFR/HER1), HER3 and HER4. Amplification of the HER2 gene and/or overexpression of its protein product have been found in up to $25 \%$ to $30 \%$ of human breast cancers ${ }^{2,3}$ and have been shown to be associated with poorer prognosis on multivariate analyses. ${ }^{4,5}$ In an effort to improve the outcomes of patients with HER2 positive (HER2+) breast cancer, research has focused on developing therapies directed to the HER 2 receptor and its pathway. These therapies, which include the monoclonal antibody trastuzumab and the small molecule tyrosine kinase inhibitor lapatinib, as well as other novel and emerging strategies, will be discussed in this review. The mechanisms of action of the therapies discussed in this review are summarized in Table 1.

\section{Trastuzumab}

The initial HER2 targeting antibody was a murine monoclonal antibody targeting the extracellular domain IV of the receptor (muMAb 4D5), derived by immunizing mice with cells that expressed high levels of the HER2 protein product. ${ }^{6}$ In order to
Conleth Murphy

Breast Cancer Medicine Service,

Sloan-Kettering Cancer Center,

1275 York Avenue, New York,

NY 10065, USA

Tel +I 9175208833

Email murphyc@mskcc.org
(C) 2009 Murphy and Modi, publisher and licensee Dove Medical Press Ltd. This is an Open Access article which permits unrestricted noncommercial use, provided the original work is properly cited. 
decrease the risks associated with the development of human antimouse antibodies, a humanized version (rhuMAb HER2 or trastuzumab) was engineered by inserting the antigenbinding residues of 4D5 into a cloned human immunoglobulin $\mathrm{G}(\mathrm{IgG})$ framework. ${ }^{7}$ The mechanisms through which trastuzumab exerts its effects in vivo are not completely understood but are likely to include antibody-dependent cellular cytotoxicity (ADCC), ${ }^{8}$ disruption of critical downstream proliferative signaling pathways and inhibition of cell cycle progression, ${ }^{9}$ and antiangiogenic effects. ${ }^{10}$ Some evidence suggests that downregulation of surface HER2 protein by endocytosis and degradation is a further mechanism of action of trastuzumab, ${ }^{11}$ although data are conflicting. ${ }^{12}$

Following extensive preclinical testing, a phase II study evaluated trastuzumab single agent therapy in 46 women with pretreated HER2+ metastatic breast cancer (MBC) ${ }^{13}$ HER2 positivity was defined as those tumors in which at least $25 \%$ of cells exhibited characteristic membrane staining for the HER2 receptor. Based on preclinical data, trastuzumab was administered as a loading dose of $250 \mathrm{mg}$ followed by $100 \mathrm{mg}$ weekly for 10 weeks. The overall response rate (ORR) was $11.6 \%$ and an additional $37 \%$ of patients achieved minimal responses or stable disease. This study provided the first clinical evidence for activity with an HER2 targeted therapy in breast cancer.

Subsequent larger multicenter phase II studies delivering weekly trastuzumab monotherapy for metastatic disease have confirmed these results. Cobleigh et al treated 222 women with HER2+ pretreated MBC with trastuzumab $4 \mathrm{mg} / \mathrm{kg}$ loading dose, followed by weekly infusions of $2 \mathrm{mg} / \mathrm{kg}{ }^{14}$ HER2 positivity was defined in terms of the protein product by central laboratory immunohistochemistry (IHC) staining of $2+$ or $3+$. The overall response rate in these pretreated patients was $15 \%$, with a duration of response of 9.1 months.

Vogel et al enrolled 114 patients with previously untreated HER2+ MBC, randomizing patients between two dose schedules of trastuzumab: a $4 \mathrm{mg} / \mathrm{kg}$ loading dose followed by $2 \mathrm{mg} / \mathrm{kg}$ weekly as in the previous study, and a higher dose schedule using $8 \mathrm{mg} / \mathrm{kg}$ as a loading dose followed by $4 \mathrm{mg} / \mathrm{kg}$ weekly. ${ }^{15}$ The ORR in these patients was $26 \%$ with no difference in outcomes between the two dose schedules. Notably, the response rate for tumors staining $3+$ by IHC was $35 \%$, with no responses seen in the group staining 2+. Retrospective HER2 gene amplification analysis by fluorescence in situ hybridization (FISH) was performed on a subset of women in this study: response rates of $34 \%$ were seen in the FISH-positive women compared with 7\% in the FISH-negative women.

\section{Pivotal trastuzumab trials for metastatic disease}

The landmark phase III trial which led to the approval of trastuzumab for metastatic disease in combination with chemotherapy, enrolled 469 women with HER 2+ MBC who were randomized to standard first-line chemotherapy or chemotherapy plus trastuzumab. ${ }^{16}$ HER2 positivity was defined

Table I Mechanisms of action of agents discussed

\begin{tabular}{|c|c|c|}
\hline Drug & Drug type & Mechanism of action \\
\hline Trastuzumab & $\begin{array}{l}\text { Monoclonal antibody against } \\
\text { HER extracellular domain IV }\end{array}$ & $\begin{array}{l}\text { Antibody-dependent cell-mediated cytotoxicity }{ }^{8} \\
\text { Disrupts downstream proliferative signaling pathways } \\
\text { Inhibits cell cycle progression } \\
\text { Antiangiogenic effects } \\
\text { Endocytosis and degradation of HER2 }^{11}\end{array}$ \\
\hline Lapatinib & $\begin{array}{l}\text { Small molecule tyrosine kinase inhibitor } \\
\text { of EGFR and HER2 }\end{array}$ & $\begin{array}{l}\text { Inhibits receptors' intrinsic tyrosine kinase activity }{ }^{48} \\
\text { Prevents downstream transmission of activation signal| }\end{array}$ \\
\hline Tanespimycin & Heat shock protein 90 (HSP90) inhibitor & $\begin{array}{l}\text { Inhibits HSP90's stabilizing effect on client proteins }{ }^{73-75} \\
\text { Facilitates HER2 degradation }\end{array}$ \\
\hline Pertuzumab & $\begin{array}{l}\text { Monoclonal antibody against HER2 } \\
\text { extracellular domain II }\end{array}$ & $\begin{array}{l}\text { Antibody dependent cell-mediated cytotoxicity } \\
\text { Prevents HER2 heterodimerization } \\
{ }^{87}\end{array}$ \\
\hline $\begin{array}{l}\text { Trastuzumab- } \\
\text { MCC-DMI }\end{array}$ & $\begin{array}{l}\text { Antibody drug conjugate linking } \\
\text { trastuzumab to microtubule cytotoxic }\end{array}$ & Targeted cytotoxic drug delivery ${ }^{90}$ \\
\hline Neratinib & $\begin{array}{l}\text { Irreversible pan-erbB tyrosine kinase } \\
\text { inhibitor }\end{array}$ & $\begin{array}{l}\text { Irreversibly inhibits tyrosine kinase activity of HER I, HER2 and HER } 49 \\
\text { Prevents downstream transmission of activation signal }\end{array}$ \\
\hline Everolimus & $\begin{array}{l}\text { Small molecule serine-threonine kinase } \\
\text { inhibitor of mTOR }\end{array}$ & Inhibits proliferative signaling pathway downstream of HER2 $2^{96}$ \\
\hline
\end{tabular}


by IHC staining of $2+$ or $3+$, rather than by gene amplification. Standard chemotherapy consisted of an anthracycline (doxorubicin or epirubicin) plus cyclophosphamide for those patients who had not been exposed to anthracyclines in the adjuvant setting, or paclitaxel for those who had previously received anthracyclines. Additionally, patients receiving chemotherapy alone could cross over to receive trastuzumab at the time of progression. Chemotherapy was administered every 3 weeks for 6 cycles (with an option of continuing at the investigator's discretion) and trastuzumab was given weekly until progression of disease. The primary endpoint of the trial was time to progression (TTP), and this was prolonged from 4.6 to 7.4 months with the addition of trastuzumab to chemotherapy $(P<0.001)$; there was also an improvement in the objective response rate from $32 \%$ to $50 \%(P<0.001)$, the median duration of response from 6.1 to 9.1 months $(P<0.001)$ and a prolongation of median overall survival (OS) from 20.3 versus 25.1 months $(P=0.046)$ in favor of the trastuzumab arm. These clinical outcomes are particularly striking given that $2 / 3$ of the patients in the control arm crossed over to receive trastuzumab at the time of progression. These were the pivotal data that led to the approval of trastuzumab in combination with chemotherapy for the first line treatment of HER2+ MBC. A second randomized trial of docetaxel chemotherapy \pm trastuzumab for a comparable cohort of patients produced similar results. ${ }^{17}$

A number of phase II studies have evaluated the addition of trastuzumab to different chemotherapy agents including docetaxel, ${ }^{18}$ paclitaxel, ${ }^{19}$ vinorelbine,${ }^{20}$ capecitabine,${ }^{21-23}$ platinum agents ${ }^{24,25}$ and gemcitabine ${ }^{26}$ with ORR ranging from $20 \%$ to $68 \%$ and acceptable toxicity profiles. The definition of HER2 positivity varied for these trials, with some using IHC staining of $2+/ 3+$ as eligibility criteria, and others stipulating IHC staining of 3+ and/or FISH positivity. Overall, these combinations represent viable salvage treatment options for patients with advanced stage HER2+ MBC.

\section{Trastuzumab beyond progression?}

A critical issue regarding the continued use of trastuzumab in the face of disease progression has only recently been addressed by prospective randomized data. Von Mincwitz and colleagues conducted a phase III trial of capecitabine vs capecitabine plus trastuzumab in patients with HER2+ MBC progressing on trastuzumab-containing therapy. ${ }^{27}$ Although accrual to this trial was halted early on the advice of an independent data monitoring committee with only 156 of a planned 482 patients enrolled, there was a significant improvement in progression-free survival (PFS) from
5.6 months to 8.2 months in the trastuzumab arm at a median follow-up of 15.6 months $(P=0.03)$.

These were the first randomized data to show a benefit for the continuation of trastuzumab in spite of disease progression, lending credence to an approach that was previously widely adopted in common practice for the treatment of patients with HER2+ disease.

\section{Adjuvant trastuzumab trials}

The improvement in outcomes resulting from the addition of trastuzumab to chemotherapy in the metastatic setting led to its investigation as an adjunct to chemotherapy in the adjuvant arena. Four large multicenter randomized trials accrued thousands of patients, and reported interim outcome analyses in 2005: NSABP B-31, NCCTG N9831, HERA and BCIRG $006 .{ }^{28-30}$ In addition, two smaller studies designed to compare different chemotherapy regimens incorporated a randomization to trastuzumab for HER2+ patients, and reported outcomes in subsequent years: FinHER and PACS 04. ${ }^{31,32}$ The study designs of these trials are summarized in Figure 1.

In the B-31 and N9831 trials, patients with early stage HER 2+ breast cancer were all treated with a standard North American adjuvant chemotherapy regimen of 4 cycles of doxorubicin and cyclophosphamide (AC) followed by paclitaxel $(\mathrm{T})$; half of these patients were randomized to additionally receive trastuzumab therapy for 1 year. Because of similarities in the design and patient populations of the two studies, a joint analysis of the data was undertaken, with the primary endpoint of evaluating disease-free survival (DFS). ${ }^{29}$ In both trials trastuzumab was initiated with the taxane component of chemotherapy in the investigational arm (a third arm of the N9831 study deferred trastuzumab until after the taxane). A combined total of 1833 patients were randomized to trastuzumab, with a control population of 1843 patients. Lymph-node positive patients only were enrolled, apart from 191 'high-risk' node-negative patients who were accrued to N9831 after a protocol amendment in May 2003. At a median follow-up of 2 years (2.4 years for B-31 and 1.5 years for N9831) there was a significant improvement in DFS for patients receiving trastuzumab, with a hazard ratio (HR) for first events of 0.48 (95\% CI 0.39-0.59; $P<0.0001)$. In addition, there was a $33 \%$ reduction in the risk of death in this group (HR 0.67, 95\% CI 0.48-0.93; $P=0.015$ ).

Simultaneously, a European multi-center trial (HERA) was evaluating the use of trastuzumab after chemotherapy in over 5000 HER2+ patients. ${ }^{28}$ While the majority of patients in this study were also node-positive, approximately one third of the patients had node-negative tumors measuring at least $1 \mathrm{~cm}$. 


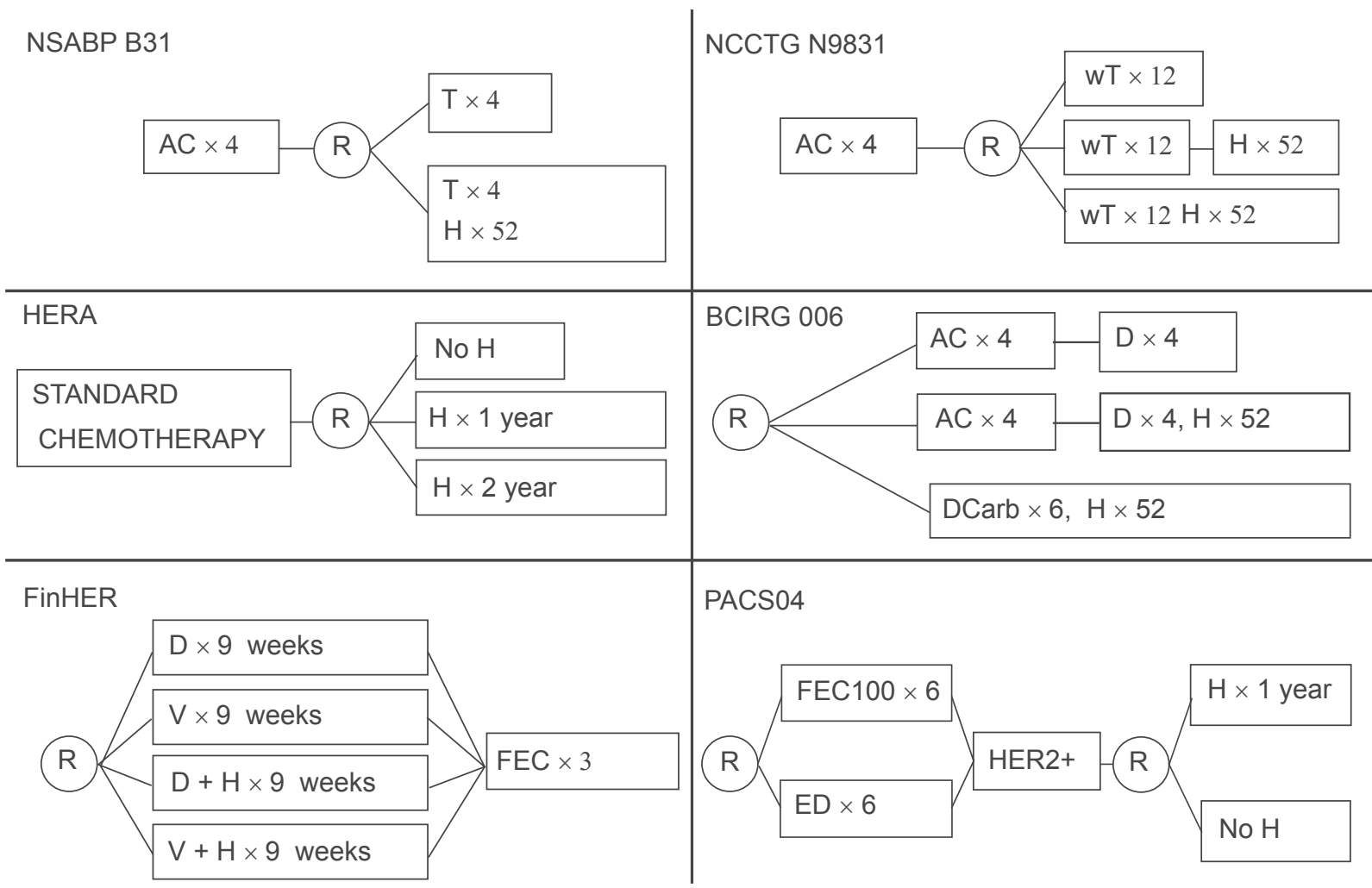

Figure I Study designs of the adjuvant trastuzumab trials.

Abbreviations: AC, doxorubicin $60 \mathrm{mg} / \mathrm{m}^{2}$ plus cyclophosphamide $600 \mathrm{mg} / \mathrm{m}^{2}$ every 3 weeks; T, paclitaxel I75 mg/m² every 3 weeks; H, trastuzumab $4 \mathrm{mg} / \mathrm{kg}$ loading dose followed by $2 \mathrm{mg} / \mathrm{kg}$ weekly $\times 5 \mathrm{I}$ weeks; wT, weekly paclitaxel $80 \mathrm{mg} / \mathrm{m}^{2}$; D, docetaxel $100 \mathrm{mg} / \mathrm{m}^{2}$ every 3 weeks; DCarb, docetaxel $75 \mathrm{mg} / \mathrm{m}^{2}+$ carboplatin AUC6 every 3 weeks; V, vinorelbine $25 \mathrm{mg} / \mathrm{m}^{2}$ Day I, 8 and 15 every 3 weeks; FEC, fluorouracil $600 \mathrm{mg} / \mathrm{m}^{2}$, epirubicin $60 \mathrm{mg} / \mathrm{m}^{2}$ plus cyclophosphamide $600 \mathrm{mg} / \mathrm{m}^{2}$ every 3 weeks; FECl00, fluorouracil $500 \mathrm{mg} / \mathrm{m}^{2}$, epirubicin $100 \mathrm{mg} / \mathrm{m}^{2}$ plus cyclophosphamide $500 \mathrm{mg} / \mathrm{m}^{2}$ every 3 weeks; ED, epirubicin $75 \mathrm{mg} / \mathrm{m}^{2}$ plus docetaxel $75 \mathrm{mg} / \mathrm{m}^{2}$ every 3 weeks.

The investigators randomized patients to either 1 or 2 years of trastuzumab versus observation after chemotherapy. A single chemotherapy regimen was not selected, but patients must have received a minimum of 4 cycles of predefined adjuvant and/or neoadjuvant therapy. The first interim analysis after only 1 year of median follow-up demonstrated a statistical improvement in DFS among patients receiving 1 year of trastuzumab compared with observation, with a HR of 0.54 (95\% CI 0.43-0.67; $P<0.0001)$. At the second interim analysis with a median follow-up of 23.5 months, there was a statistically significant $34 \%$ reduction in the risk of death (HR $0.66,95 \%$ CI $0.47-0.91$; $P=0.0115) .{ }^{33}$ Efficacy results for the 2-year trastuzumab arm are keenly awaited and are expected in 2009.

The BCIRG 006 study randomized 3222 HER2+ patients to 3 arms: doxorubicin and cyclophosphamide (AC) for 4 cycles followed by docetaxel for 4 cycles; AC for 4 cycles followed by docetaxel for 4 cycles plus trastuzumab for 1 year; and a third novel arm lacking an anthracyline, of docetaxel plus carboplatin and trastuzumab, with trastuzumab continuing for 1 year. Based on preclinical data suggesting a synergistic effect of trastuzumab with platinum compounds, this study incorporated this third arm without anthracyclines to try and minimize cardiac risk. At an interim analysis performed at 36 months of follow-up, the results demonstrated a significant improvement in DFS and OS for both trastuzumab containing arms compared to chemotherapy alone. ${ }^{34}$ The HR for DFS in the anthracycline-containing trastuzumab and nonanthracycline arms compared to the control arm were 0.61 and 0.67 , respectively; for OS, the HRs were 0.59 and 0.66 , respectively. All results were statistically significant. Most importantly, the incidence of grade $3 / 4$ cardiac toxicity with the novel nonanthracycline arm was similar to the control arm $(0.4 \%$ vs $0.3 \%)$ and less than that of the anthracycline plus trastuzumab arm $(2 \%)$. This was the fourth trial to confirm a significant reduction in the risk of recurrence with the addition of trastuzumab to chemotherapy for HER2+ early stage breast cancer, with the additional advantage of a minimally cardiotoxic chemotherapy regimen.

FinHER was a small study in which HER2+ patients (232 in total) were randomized to a notably shorter duration of 
trastuzumab therapy (9 weekly doses) in combination with either docetaxel or vinorelbine or to these chemotherapy agents alone. ${ }^{31}$ All patients then proceeded to 3 cycles of anthracycline-containing chemotherapy. This study was powered to detect a $50 \%$ to $67 \%$ improvement in recurrence-free survival in the HER2+ patients treated with trastuzumab, at a power of 0.80 . In spite of this threshold, the study achieved this aim with a HR for recurrence of 0.42 (95\% CI 0.21-0.83; $P=0.01)$ and a HR for death of 0.41 (95\% CI 0.16-1.08; $P=0.07)$. Although the study was small, it achieved similar levels of risk reduction as seen with 1 year of adjuvant trastuzumab, and raised the question of duration for the antibody in this setting. While currently 1 year of adjuvant trastuzumab remains the standard of care for HER2+ early breast cancer, results from the 2-year arm of the HERA trial and the ongoing Greek ${ }^{35}$ and French $^{36}$ studies randomizing patients to 6 vs 12 months of trastuzumab in the adjuvant setting may help define the optimal duration of treatment.

The lone trial that has not shown an advantage for trastuzumab in the adjuvant setting is the PACS 04 trial in which 528 HER2+ patients were randomized to 1 year of trastuzumab or observation after completion of adjuvant chemotherapy. ${ }^{32}$ Although there was a trend for benefit in the first 18 months, in the intent-to-treat analysis after a median follow-up of 48 months, there was no significant difference observed in either the DFS or OS between the trastuzumab and the observation arms. The small study size, statistical power, and sequential versus concurrent administration of the trastuzumab in this trial have been raised as possible explanations for this negative result; additional studies and further followup of the 4 large randomized trials and other ongoing studies may help to place these provocative data in context.

\section{Trastuzumab toxicity}

It was not immediately apparent from the early clinical trials with trastuzumab that cardiac toxicity would be a major concern with this agent. Rates of cardiac dysfunction in the two major studies of trastuzumab monotherapy (as defined by congestive heart failure, cardiomyopathy or left ventricular ejection fraction (LVEF) decline $>10 \%$ points) were relatively modest at $2 \%$ to $4.7 \% .^{14,15}$ However, in the landmark phase III trial with chemotherapy, the use of trastuzumab concurrently with anthracyclines appeared to dramatically increase the rate of cardiac dysfunction, from $8 \%$ in the anthracycline chemotherapy group to $27 \%$ in the anthracycline and trastuzumab group. ${ }^{16}$ Accordingly, concurrent use of anthracyclines and trastuzumab is not advised, apart from the neoadjuvant setting where there is limited experience demonstrating safety and efficacy for concurrent administration.

Given the potential for cardiac dysfunction, there was rigorous cardiac monitoring as part of the adjuvant trastuzumab trials which has allowed for prospective monitoring and evaluation of this toxicity. After 5 years of follow-up, there was a $4 \%$ incidence of class III/IV congestive heart failure $(\mathrm{CHF})$ or cardiac death in the patients receiving trastuzumab on the adjuvant B31 trial. ${ }^{37}$ A detailed cardiac safety analysis of this trial revealed that trastuzumab was discontinued for asymptomatic decreases in LVEF in 14\% of evaluable patients. ${ }^{38}$ Of those who had a repeat LVEF assessment at 6 months after discontinuation, only $25 \%$ had an LVEF value persistently below 50\%. Similarly, in BCIRG $00617.3 \%$ of patients receiving AC-TH sustained a $>10 \%$ decline in LVEF relative to baseline, with $26 \%$ of patients who had repeat assessments after 42 days demonstrating persistent declines. ${ }^{37}$ Hence, the majority of patients will experience improved cardiac function with or without appropriate medications for $\mathrm{CHF}$, suggesting a reversibility to the cardiac dysfunction induced by trastuzumab and distinguishing it from the damage imposed by anthracyclines which is largely irreversible. ${ }^{39}$

And while the underlying mechanism of trastuzumabinduced cardiotoxicity is not well understood, animal studies have suggested an important role for HER2 signaling pathways in embryonic heart development, and in the prevention of dilated cardiomyopathy. ${ }^{40}$ Thus signaling through the HER2 pathway may be important for cardiac myocyte survival during periods of stress. In the absence of preventative measures, cardiac monitoring is imperative for patients on trastuzumab, particularly in the adjuvant setting where left ventricular function must be monitored every 3 to 4 months.

Beyond cardiac toxicity, trastuzumab is rarely associated with typical chemotherapy-type side effects. The most common noncardiac toxicity is infusion reactions, which were seen in $25 \%$ of patients during the first infusion in the landmark study in combination with chemotherapy, and generally did not recur on subsequent infusions. ${ }^{16}$ Grade 3 and 4 infusion reactions were not seen in the adjuvant trastuzumab studies, perhaps due to careful pre-medication and monitoring of patients. A rare but notable toxicity encountered in the NSABP B31 and NCCTG N9831 studies was interstitial pneumonitis, which was fatal in 1 patient in each study. ${ }^{29}$ Additional adverse events encountered with trastuzumab alone or with chemotherapy in clinical trials for early and advanced breast cancer include arthralgia, myalgia, fatigue, dyspnea, nail 
changes, rash, headache, insomnia, thrombosis/embolism, and diarrhea, ${ }^{41}$ with grades 3 and 4 of any of these toxicities occurring in under $1 \%$ of patients receiving trastuzumab after adjuvant chemotherapy in the HERA study. ${ }^{28}$ The incidence of grade 4-5 neutropenia was increased from $0.7 \%$ to $2 \%$ when trastuzumab was added to chemotherapy in the NCCTG N9831 adjuvant study, and grade 3-5 infections/febrile neutropenia were increased from $1.4 \%$ to $3.3 \% .{ }^{41}$ Anemia is also seen with trastuzumab, with $<1 \%$ of patients receiving trastuzumab as a single agent for $\mathrm{MBC}$ experiencing grade 3 anemia. ${ }^{14}$ Rare cases of glomerulopathy causing nephrotic syndrome have also been reported in the postmarketing setting. ${ }^{41}$

\section{Trastuzumab: mechanisms of resistance}

In spite of its success in the treatment of both early and advanced stage HER2+ breast cancer, a proportion of patients who receive trastuzumab-containing adjuvant therapy will relapse and nearly all patients receiving trastuzumab for metastatic disease will experience progression. A number of potential mechanisms underlying trastuzumab resistance have been proposed and include inhibition of the interaction of trastuzumab with its target protein by increased expression of the membrane glycoprotein MUC4 ${ }^{42}$ or shedding of the extracellular domain of the receptor leaving behind the truncated form of the receptor (p95) which retains kinase activity but cannot bind to trastuzumab. In an analysis of 46 patients with HER2+ MBC, p95 expression in the tumors was associated with clinical trastuzumab resistance. ${ }^{43}$ Cross talk or heterodimerization between HER2 and IGF-1 ${ }^{42}$ or HER family members ${ }^{44}$ may be another potential mechanism of resistance to trastuzumab. In these cases, signaling through other growth factor receptors may lead to the same downstream activation of the PI3K pathway resulting in cell proliferation. Looking at the pathway itself, activating mutations AKT and decreased levels of PTEN expression have also has been associated with trastuzumab resistance. ${ }^{45,46}$ Other potential mechanisms of resistance explored in preclinical models include downregulation of a cyclin-dependent kinase (p27 kipl), a downstream effector of multiple growth factor receptor pathways, ${ }^{47}$ and transcriptional upregulation of HER2 gene expression. ${ }^{48}$ Ultimately, a clear and better understanding of the molecular mechanisms of resistance to trastuzumab will allow clinicians to identify the optimal treatment strategies for individual patients with HER2+ disease.

\section{Lapatinib}

As described above, de novo and acquired trastuzumab resistance are major clinical problems. Hence, new agents targeting this growth signaling pathway are urgently needed.
The first of this new generation of HER2 targeting drugs, lapatinib $\left(\right.$ Tykerb $^{\circledR} /$ Tyverb $^{\circledR}$ ), was approved for the treatment of HER2+ MBC by the US Food and Drug Administration (FDA) in 2007. Lapatinib, previously known as GW572016, is a reversible small-molecule tyrosine kinase inhibitor which targets both HER2 and EGFR tyrosine kinases and has the advantage of being administered orally. In vitro studies demonstrated superior growth-inhibitory effects for this molecule in HER2 and EGFR overexpressing cell lines. ${ }^{49}$ Additionally, lapatinib can inhibit the growth of HER2+ human breast cancer cells that are resistant to the effects of trastuzumab, ${ }^{50}$ as well as enhance the activity of anti-HER2 antibodies when used together. ${ }^{51}$ Activity in vivo was confirmed in HER2+ human breast carcinoma xenografts in mice. ${ }^{49,52}$ Inhibition of activation of downstream signaling molecules was also demonstrated in human tumor xenograft models. $^{53}$

\section{Lapatinib trials}

The first human studies of lapatinib were two phase I pharmacokinetic studies testing low doses of the drug in healthy subjects. Peak serum concentrations were detected at a median of 3 hours after the dose, with steady state concentrations being achieved at 6 to 7 days. The most common toxicities encountered in these healthy volunteers were headache, diarrhea, rash, cold symptoms, gastrointestinal symptoms and elevation of liver enzymes. ${ }^{54}$ A subsequent phase I study evaluated the safety of lapatinib in 64 patients with solid tumors (including 4 with breast cancer). ${ }^{55-57}$ 39 patients received once daily dosing at doses ranging from 175 to $1800 \mathrm{mg}$ daily. Twenty-five patients received doses of $500 \mathrm{mg}, 750 \mathrm{mg}$ or $900 \mathrm{mg}$ twice daily. The most commonly encountered toxicities in all cohorts were transient grade 1-2 rash, diarrhea, nausea/vomiting, fatigue and anorexia. Grade 3 diarrhea was seen in 2 of 6 patients at $900 \mathrm{mg}$ bid, requiring dose reductions. A complete tumor remission was seen in 1 patient with EGFR overexpressing squamous cell carcinoma of the head and neck; in addition 22 patients experienced stable disease with a median duration of 4 months. All of the patients who continued therapy beyond 4 months were on daily doses of at least $1200 \mathrm{mg}$. The authors concluded that daily lapatinib dosing was well tolerated with evidence of clinical activity. Another phase I study evaluated 5 once daily dose cohorts in a more selected population of 67 patients with EGFR and/or HER2 overexpressing metastatic cancers. ${ }^{58}$ Daily doses ranged from $500 \mathrm{mg}$ to $1600 \mathrm{mg}$. Lapatinib was generally well tolerated, with the most common toxicities being diarrhea in 
$42 \%$ and rash in $31 \%$. The incidence of diarrhea was found to be increased with increasing dose. There were 4 partial responses (PRs), all of which were documented in patients with trastuzumab-resistant MBC (including 2 patients with inflammatory breast cancer).

A number of phase II studies followed, evaluating lapatinib monotherapy in HER2+ patients with advanced breast cancer. In the first of these, 140 patients with HER2-overexpressing and 89 with HER2 negative advanced breast cancer were treated with lapatinib $1500 \mathrm{mg}$ daily. ${ }^{59}$ This was a heavily pretreated population, $76 \%$ of patients having received 4 or more lines of prior chemotherapy. There were no responses seen in the HER2 - cohort. The HER2+ cohort appeared to derive a slight benefit, however, with an ORR of $4.3 \%$ as determined by the investigators, and $1.4 \%$ as determined by the independent review committee. In a phase II study of HER2+ patients only, 78 patients who had progressed on prior trastuzumab therapy were randomly allocated to lapatinib as a single agent at $1250 \mathrm{mg}$ or $1500 \mathrm{mg}$ daily. ${ }^{60}$ The ORR was again relatively low in this study, at $7.7 \%$ by investigator interpretation and $5.1 \%$ as per the independent review committee. The time to progression was 15.3 weeks.

The EGF20009 study evaluated lapatinib monotherapy in a first-line setting. ${ }^{61} 138$ patients who were previously untreated in the metastatic setting were randomized to $1500 \mathrm{mg}$ orally daily or $500 \mathrm{mg}$ twice daily. All patients were required to be HER2+ by FISH analysis; HER2 protein expression was not evaluated. No patients had received prior trastuzumab. Results were more impressive in this cohort of patients, with an ORR of $24 \%$. By indirect comparison this result compares favorably with the ORR of $34 \%$ in the subgroup of women with HER2 FISH-amplified MBC treated in a phase II study of upfront trastuzumab monotherapy. ${ }^{15}$ The PFS rate at 4 months in the lapatinib study was $63 \%$, and $43 \%$ at 6 months. ${ }^{61}$ There were no significant differences in outcomes between the two dosing groups. The common toxicities reported by investigators to be lapatinib-related were diarrhea (36\%), rash (27\%), pruritus (18\%) and nausea (10\%).

\section{Randomized lapatinib trials}

Two large randomized phase III studies investigated the addition of lapatinib to chemotherapy regimens for advanced breast cancer. EGF100151 was an open-label study which enrolled patients with HER2+ advanced breast cancer who had received previous anthracycline, taxane and trastuzumab. ${ }^{62,63}$ The control arm received capecitabine $2500 \mathrm{mg} / \mathrm{m}^{2}$ daily for 14 days on a 21 -day cycle. The investigational arm received a lower daily dose of capecitabine $\left(2000 \mathrm{mg} / \mathrm{m}^{2}\right)$ on the same schedule, with oral lapatinib at $1250 \mathrm{mg}$ daily continuously. Study enrollment began on March 29th, 2004. A data lock-in was performed in November 2005, based on a pre-specified event threshold. The outcomes of the 324 women who had been accrued to date were subject to an interim analysis, following which the data safety monitoring committee recommended reporting the study results and offering lapatinib to women who had been receiving capecitabine monotherapy on the control arm. This was based on a significant improvement in the primary study endpoint of time to progression (TTP), with a HR of $0.49(95 \% \text { CI } 0.34-0.71, P<0.001)^{62}$ with the combination treatment; additionally TTP was 8.4 months versus 4.4 months in favor of the lapatinib arm. There were no significant differences in response rate or survival. An updated analysis included all 399 women who had entered the trial before accrual was closed in April 2006. In this analysis, TTP remained significantly prolonged in the lapatinib combination group (6.2 vs 4.3 months, HR 0.57; 95\% CI 0.43-0.77, $P<0.001) ;{ }^{63}$ the ORR was also significantly higher for the combination $(24 \%$ vs $14 \%, P=0.017)$. In an exploratory analysis, the frequency of central nervous system (CNS) as site of first progression was lower in the lapatinib arm (4 vs $13, P=0.045$ ). Not surprisingly given the individual toxicity profiles of capecitabine and lapatinib, diarrhea was the most common toxicity for the combination, occurring in $60 \%$ of patients. $12 \%$ experienced grade 3 diarrhea, and $1 \%$ grade 4 . Other toxicities commonly encountered in the combination arm were hand foot syndrome (49\%), rash (27\%), nausea (44\%), vomiting (26\%) and fatigue (18\%). The results of this trial were the basis for the approval of lapatinib by the FDA.

The other phase III study of lapatinib in combination with chemotherapy (EGF30001) was a randomized, placebocontrolled study that examined the impact of adding lapatinib to paclitaxel as first-line therapy of advanced breast cancer. ${ }^{64}$ In contrast to the capecitabine study, HER2 negative and HER2 uncharacterized patients were enrolled onto this study. No patients had known HER2+ disease at study entry, although it was expected that a proportion would turn out to be HER2+ on testing. The paclitaxel dose was $175 \mathrm{mg} / \mathrm{m}^{2}$ by intravenous infusion every 3 weeks, and patients were randomized to either lapatinib $1500 \mathrm{mg}$ or placebo daily. In the intent-to-treat population of 579 patients, the response rate was higher for the combination arm (35\% vs $25 \%$, odds ratio $=1.7 ; 95 \% \mathrm{CI}, 1.1-2.4 ; P=0.008)$; however there were no significant difference in TTP, event-free survival (EFS) or OS. 86 patients on this study were found to have HER2+ MBC and the addition of lapatinib for this group was associated 
with a longer median TTP of 36.4 vs 25.1 weeks, with a HR for progression of $0.53(95 \% \mathrm{CI}, 0.31-0.89, P=0.005)$. EFS, ORR and clinical benefit rate (CBR) were also significantly better with combined therapy in the HER2+ subpopulation. Conversely, there was no advantage in any of the efficacy endpoints in the HER2 negative patients. The addition of lapatinib to paclitaxel was associated with significantly higher rates of rash, diarrhea, mucositis and vomiting. Furthermore, there was an increased rate of fatal adverse events in the paclitaxel/lapatinib group ( 8 patients, $2.7 \%$ ) compared to the paclitaxel/placebo group ( 2 patients, $0.6 \%$ ). The fatal adverse events in the lapatinib group included three cases of sepsis associated with diarrhea, mostly occurring early in the course of the trial before the investigators instituted aggressive measures to proactively manage diarrhea. The results of this study showed that HER2 negative unknown patients do not benefit from the addition of lapatinib to paclitaxel.

Lapatinib has also been combined with endocrine therapy for $\mathrm{MBC}$, based on the pre-clinical evidence for cross-talk between growth factor receptor (such as HER2 and EGFR) and hormone receptor pathways as a potential mechanism for endocrine resistance. ${ }^{65}$ Results of the EGF30008 study were presented at the San Antonio Breast Cancer Symposium in December 2008. ${ }^{66}$ This study randomized 1208 patients with $\mathrm{MBC}$ to letrozole $2.5 \mathrm{mg}$ daily with either lapatinib $1500 \mathrm{mg}$ daily or placebo. Of the 219 patients in this study who had HER2+ disease, the addition of lapatinib to letrozole improved PFS from 3.0 months to 8.2 months $(\mathrm{HR}=0.71$; 95\% CI, 0.53-0.96, stratified $\log$-rank $P=0.019$ ) and response rates (from $28.7 \%$ to $47.7 \%$ ). No effect on PFS or response rate was seen in the HER2 negative patients although an exploratory Cox regression analysis suggested a PFS benefit for lapatinib, in patients with recent tamoxifen treatment (representing endocrine resistance) as an important covariate. The combination treatment was well tolerated, with no unexpected toxicities and a low rate of asymptomatic LVEF decline (2\%). A similar result was observed in the TAnDEM trial which randomized 208 patients with HER2+ MBC to first-line therapy with anastrozole \pm trastuzumab. ${ }^{67}$ Outcomes were superior with the combination arm in terms of PFS (4.8 vs 2.4 months, $P=0.0016)$, ORR (20.3\% vs $6.8 \%$, $P=0.018)$, and CBR (42.7\% vs $27.9 \%, P=0.026)$. Although there was a trend in OS favoring the combination arm, it was not statistically significant (28.5 vs 23.9 months, $P=0.325$ ). About $70 \%$ of the patients in the anastrozole only group crossed over to receive trastuzumab upon progression of disease, and the OS was the same in this group as in those who received the upfront combination.
O'Shaughnessy et al have reported results from a randomized trial of lapatinib alone versus lapatinib plus trastuzumab in patients with HER2+ MBC who progressed on trastuzumab. ${ }^{68}$ Patients were treated with either lapatinib at $1500 \mathrm{mg}$ daily or lapatinib at $1000 \mathrm{mg}$ daily plus weekly conventional trastuzumab. If patients progressed on the lapatinib monotherapy arm, they could cross over to the lapatinib plus trastuzumab regimen. 296 pts were randomized and results revealed a progression free survival advantage in favor of the combination of 12 weeks versus 8.4 weeks $(P=0.029)$ and an improvement in the clinical benefit rate of 25.2 vs 13.2 weeks $(P=0.02)$; response rate and overall survival were similar in both arms. Both treatment regimens were generally well tolerated. The improved clinical outcome achieved with the combination of lapatinib and trastuzumab in patients progressing on trastuzumab-based therapy further confirms the role for continued trastuzumab in spite of progression and additionally supports a role for dual modality HER2 inhibition.

\section{Lapatinib in CNS disease}

Given its large molecular size, trastuzumab is unable to cross the blood-brain barrier (BBB) effectively. This is of particular concern, given the frequency of CNS metastases in HER2+ MBC. In a report by Bendell et al 34\% of patients with HER2+ MBC developed CNS metastases at a median of 6 months from the initiation of trastuzumab therapy for metastatic disease, with $50 \%$ of these patients having stable or responding disease outside the CNS at the time. ${ }^{69}$ Furthermore, half of the patients with CNS metastases died as a result of progressive CNS disease, rather than systemic disease. Conversely, lapatinib has been shown to reduce the formation of large brain metastases in a mouse xenograft model using a trastuzumab-resistant HER2+ MBC line. ${ }^{70}$ A phase II study of lapatinib monotherapy in 241 patients with progressive brain metastases after trastuzumab and radiotherapy demonstrated a disappointing response rate of $2.6 \%,{ }^{71}$ however the authors noted that $18 \%$ of patients were progression-free at 16 weeks, and reported some favorable volumetric changes in CNS disease based on exploratory analysis. Further prospective evaluation of the role of lapatinib in the prevention of CNS disease will be explored in the adjuvant studies of lapatinib that are currently underway.

\section{Adjuvant lapatinib trials}

The Adjuvant Lapatinib and/or Trastuzumab Treatment Optimization (ALTTO) study is an ongoing, four arm randomized study evaluating lapatinib (arm A), trastuzumab (arm B), 
trastuzumab followed by lapatinib (arm C), or concurrent treatment with both agents (arm D) for early stage HER2+ breast cancer. ${ }^{72}$ Two trial designs are available: In design 1 , patients will be randomized to one of the four anti-HER2 treatment strategies described above after completing adjuvant anthracycline-containing chemotherapy; in design 2 , the anti-HER2 therapy will commence concurrently with paclitaxel, after surgery and anthracycline-based chemotherapy. A sister trial, neo-ALTTO, will test the anti-HER2 regimens in the neoadjuvant setting. An unexpected toxicity of grade 3 diarrhea seen with the concurrent paclitaxel/trastuzumab/ lapatinib combination was observed in a pilot study of this combination following dose-dense AC, where lapatinib was given at a dose of $1000 \mathrm{mg}$ daily. This combination was not feasible with $34 \%$ of patients requiring a lapatinib dosereduction, predominantly due to grade 3 diarrhea $^{73}$. These findings led to a modification of the lapatinib dose on the corresponding arm of ALTTO trial (design 2, arm D).

\section{Other HER2 therapies HSP90 inhibitors}

Heat shock protein 90 (HSP90) is a molecular chaperone which plays an important role in the maturation and conformational stabilization of a number of cellular 'client' proteins. When HSP90 is inhibited its clients are rendered unstable and ultimately they undergo degradation in the proteasome. ${ }^{74-76}$ One of the most sensitive client proteins of Hsp90 inhibition is HER2. ${ }^{77,78}$

Tanespimycin (17AAG, 17-allylamino-17-demethoxygeldanamycin), a geldanamycin derivative, is the lead Hsp90 inhibitor in clinical development and inhibits Hsp90 function in tumors in a variety of murine models, and it has now been extensively studied as a single agent in patients with solid tumors in early phase clinical trials. ${ }^{79-85}$ A phase I study of the combination of tanespimycin and trastuzumab enrolled 25 patients with advanced solid tumors, with 15 of these patients having HER2+ MBC. ${ }^{86}$ Tanespimycin was administered on a weekly schedule in escalating dose cohorts in combination with weekly conventional trastuzumab. The recommended phase 2 dose for tanespimycin was determined to be $450 \mathrm{mg} / \mathrm{m}^{2}$ weekly. The most commonly encountered toxicities were grade 1-2 vomiting, diarrhea, headache, fatigue and anorexia. Of note, no significant cardiotoxicity was encountered. Tumor regressions were observed in 5 patients, all of whom had HER2+ MBC previously progressing on trastuzumab. These results led to a phase 2 study of this combination in patients with HER2+ MBC progressing on one line of trastuzumab-based therapy.
When interim data from this study was presented at the annual meeting of the American Society of Clinical Oncology (ASCO) 2008, a total of 31 patients had been enrolled, with 27 of these evaluable for response. ${ }^{87}$ There were 7 patients with RECIST-defined partial responses (one unconfirmed) and an additional 5 patients with objective tumor regression measuring between $20 \%$ to $29 \%$ reduction in tumor burden; 5 patients also had disease stabilization for $>4$ months. The overall response rate was $26 \%$ with a clinical benefit rate of $63 \%$. Based on these clinical results, the combination of tanespimycin plus trastuzumab is highly active for trastuzumab-treated HER2+ MBC. A number of other HSP90 inhibitor compounds are also undergoing clinical evaluation for HER2+ MBC.

\section{Other HER2 antibodies}

Pertuzumab (Omnitarg ${ }^{\circledR}$; Genentech) is a humanized monoclonal antibody that binds to the extracellular domain II of the HER2 receptor, preventing receptor dimerization and ligand-activated signaling with other growth factor receptors, including other HER family members. ${ }^{88}$ In a phase II study of pertuzumab plus trastuzumab, 66 patients with HER2+ MBC progressing on trastuzumab were enrolled and treated with 3-weekly pertuzumab and weekly or 3-weekly trastuzumab. ${ }^{89}$ Interim results presented at the ASCO 2008 meeting revealed a RR of $24.2 \%$, with a CBR of $50 \%$. The most frequently encountered toxicities were grade 1 and 2 diarrhea (64\%), fatigue $(33 \%)$, nausea $(27 \%)$, rash $(26 \%)$ and headache (20\%). Only 3 patients had an LVEF which declined by more than $10 \%$ from baseline to a level less than $50 \%$. Currently a double-blind multicenter phase III study, CLEOPATRA, is randomizing patients to docetaxel plus trastuzumab versus docetaxel plus trastuzumab and pertuzumab as first-line treatment for HER2+ advanced breast cancer. ${ }^{90}$

\section{Antibody-drug conjugates}

A promising strategy involves coupling targeted antibodies with potent antitumor agents, in the form of antibody-drug conjugates (ADC). Trastuzumab-MCC-DM1 (T-DM1) is an ADC using a stable thioether linkage to couple trastuzumab to a derivative of maytansine, a chemotherapeutic agent with a microtubule-binding effect similar to vinca alkaloids. T-DM1 has antitumor activity in trastuzumab-sensitive and trastuzumab-resistant preclinical models of HER2+ breast cancer. ${ }^{91}$ A phase I study evaluated 3-weekly dosing of T-DM1 in patients with HER2+ MBC progressing on trastuzumab. ${ }^{92}$ Tumor responses were seen in 5 of 9 patients ( 4 confirmed, $44 \%$ ) at the maximally tolerated dose (MTD) of $3.6 \mathrm{mg} / \mathrm{kg}$. 
A rapidly reversible thrombocytopenia (which was grade 4 in two patients) was the dose-limiting toxicity. There was no evidence of deleterious cardiac effects. Subsequently a weekly dosing schedule achieved an MTD of $2.4 \mathrm{mg} / \mathrm{kg} .{ }^{93}$ For this schedule, 9 of 15 evaluable patients had confirmed PRs (53\%). Interim results of a phase II study of 3-weekly T-DM1 as third-line therapy for patients with HER2+ MBC progressing on prior trastuzumab, were presented in $2008 .^{94}$ 107 of 122 enrolled patients were evaluable for efficacy, with a median follow-up of 4.4 months. $55 \%$ of patients had received prior lapatinib. There was no grade 3 or 4 cardiac dysfunction reported. The confirmed response rate in evaluable patients was $27.1 \%$, and in lapatinib pretreated patients was $21.7 \%$. Further phase II studies are now underway, including a randomized comparison of T-DM1 versus the combination of trastuzumab and docetaxel as first-line therapy for HER2 $+\mathrm{MBC}^{3}$ and a randomized phase III trial of T-DM1 versus capecitabine plus lapatinib. ${ }^{32}$

\section{Other small-molecule inhibitors of HER2 signaling}

Neratinib is a low-molecular-weight pan-erbB tyrosine kinase inhibitor. In contrast to lapatinib, it irreversibly inhibits erbB1 and erbB2. It has been evaluated in trastuzumab pretreated and trastuzumab-naive patients in a phase II clinical trial. ${ }^{95}$ A daily dose of $240 \mathrm{mg}$ orally was used based on a dose-limiting toxicity of diarrhea encountered in the phase I setting. All patients had HER2 status centrally confirmed by FISH testing. The ORR was $26 \%$ for patients who had received prior trastuzumab, with a median PFS of 23 weeks. The trastuzumab-naive patients had an ORR of $56 \%$, with a median PFS of 40 weeks. The major toxicity encountered was diarrhea, occurring in $97 \%$ of patients who had received prior trastuzumab, with a grade 3 or 4 rate of $30 \%$ in this group ( $13 \%$ in the trastuzumab naive patients). This agent is now undergoing evaluation in a phase III randomized trial.

\section{mTOR inhibitors}

Loss of expression of the tumor suppressor PTEN and/or activation of the PI3K/AKT signalling proteins have been reported as possible mechanisms of resistance to trastuzumab. ${ }^{96}$ Mammalian target of rapamycin (mTOR) is a serine-threonine kinase which is a downstream component of the PTEN/PI3K pathway. Everolimus (RAD001) is an oral inhibitor of mTOR which has been evaluated with combinations of trastuzumab and various chemotherapy agents in phase I studies. Two studies reported updated analyses at SABCS 2008. Everolimus on a daily or weekly schedule was combined with weekly trastuzumab and either weekly paclitaxe ${ }^{97}$ or vinorelbine. ${ }^{98}$ Both studies enrolled patients with HER2+ MBC progressing on or after trastuzumab. The most common dose-limiting toxicities were neutropenia and stomatitis. Nine partial responses were seen among 27 evaluable patients with paclitaxel (41\%), and 6 responses among 37 patients with vinorelbine (18\%). Phase II studies are awaited.

\section{Conclusion}

Clinical studies have confirmed that trastuzumab significantly enhances the outcomes for subsets of patients with both early and late stage HER2+ breast cancer. Moreover, the HER2 receptor remains a viable target in spite of progression and is a relevant target for novel anti-HER2 strategies. Lapatinib is approved as the next line of HER2 therapy after progression on trastuzumab and other promising agents in clinical development include antibody conjugates and heat shock protein 90 inhibitors among others. Understanding the mechanisms and biochemistry of disease development and progression and targeting the specific pathways and critical proteins to the process will allow us to build on our current anticancer strategies. Trastuzumab has rightly been considered a landmark; perhaps someday it will be looked on as the first stepping stone across a wide stream.

\section{Disclosures}

The authors disclose no conflicts of interest.

\section{References}

1. Friedlander E, Barok M, Szollosi J, Vereb G. ErbB-directed immunotherapy: antibodies in current practice and promising new agents. Immunol Lett. 2008;116(2):126-140.

2. Slamon DJ, Clark GM, Wong SG, Levin WJ, Ullrich A, McGuire WL. Human breast cancer: correlation of relapse and survival with amplification of the HER-2/neu oncogene. Science. 1987;235(4785):177-182.

3. Slamon DJ, Godolphin W, Jones LA, et al. Studies of the HER$2 /$ neu proto-oncogene in human breast and ovarian cancer. Science. 1989;244(4905):707-712.

4. Seshadri R, Firgaira FA, Horsfall DJ, McCaul K, Setlur V, Kitchen P. Clinical significance of HER-2/neu oncogene amplification in primary breast cancer. The South Australian Breast Cancer Study Group. J Clin Oncol.1993;11(10):1936-1942.

5. Tandon AK, Clark GM, Chamness GC, Ullrich A, McGuire WL. HER$2 /$ neu oncogene protein and prognosis in breast cancer. J Clin Oncol. 1989; 7(8):1120-1128.

6. Fendly BM, Winget M, Hudziak RM, Lipari MT, Napier MA, Ullrich A. Characterization of murine monoclonal antibodies reactive to either the human epidermal growth factor receptor or HER2/neu gene product. Cancer Res. 1990;50(5):1550-1558.

7. Carter P, Presta L, Gorman CM, et al. Humanization of an antip185HER2 antibody for human cancer therapy. Proc Natl Acad Sci U S A.1992;89(10):4285-4289.

8. Cooley S, Burns LJ, Repka T, Miller JS. Natural killer cell cytotoxicity of breast cancer targets is enhanced by two distinct mechanisms of antibody-dependent cellular cytotoxicity against LFA-3 and HER2/neu. Exp Hematol.1999;27(10):1533-1541. 
9. Albanell J, Codony J, Rovira A, Mellado B, Gascon P. Mechanism of action of anti-HER2 monoclonal antibodies: scientific update on trastuzumab and 2C4. Adv Exp Med Biol. 2003;532:253-268.

10. Izumi Y, Xu L, di Tomaso E, Fukumura D, Jain RK. Tumour biology: herceptin acts as an anti-angiogenic cocktail. Nature. 2002;416(6878): 279-280.

11. Baselga J, Albanell J, Molina MA, Arribas J. Mechanism of action of trastuzumab and scientific update. Semin Oncol. 2001;28(5 Suppl 16): 4-11.

12. Austin CD, De Maziere AM, Pisacane PI, et al. Endocytosis and sorting of ErbB2 and the site of action of cancer therapeutics trastuzumab and geldanamycin. Mol Biol Cell. 2004;15(12):5268-5282.

13. Baselga J, Tripathy D, Mendelsohn J, et al. Phase II study of weekly intravenous recombinant humanized anti-p185HER2 monoclonal antibody in patients with HER2/neu-overexpressing metastatic breast cancer. J Clin Oncol.1996;14(3):737-744.

14. Cobleigh MA, Vogel CL, Tripathy D, et al. Multinational study of the efficacy and safety of humanized anti-HER 2 monoclonal antibody in women who have HER2-overexpressing metastatic breast cancer that has progressed after chemotherapy for metastatic disease. J Clin Oncol. 1999;17(9):2639-2648.

15. Vogel CL, Cobleigh MA, Tripathy D, et al. Efficacy and safety of trastuzumab as a single agent in first-line treatment of HER2overexpressing metastatic breast cancer. J Clin Oncol. 2002;20(3): 719-726.

16. Slamon DJ, Leyland-Jones B, Shak S, et al. Use of chemotherapy plus a monoclonal antibody against HER2 for metastatic breast cancer that overexpresses HER2. $N$ Engl J Med.2001;344(11): 783-792.

17. Marty M, Cognetti F, Maraninchi D, et al. Randomized phase II trial of the efficacy and safety of trastuzumab combined with docetaxel in patients with human epidermal growth factor receptor 2-positive metastatic breast cancer administered as first-line treatment: the M77001 study group. J Clin Oncol. 2005;23(19):4265-4274.

18. Esteva FJ, Valero V, Booser D, et al. Phase II study of weekly docetaxel and trastuzumab for patients with HER-2-overexpressing metastatic breast cancer. J Clin Oncol. 2002;20(7):1800-1808.

19. Seidman AD, Fornier MN, Esteva FJ, et al. Weekly trastuzumab and paclitaxel therapy for metastatic breast cancer with analysis of efficacy by HER2 immunophenotype and gene amplification. J Clin Oncol. 2001;19(10):2587-2595.

20. Burstein HJ, Harris LN, Marcom PK, et al. Trastuzumab and vinorelbine as first-line therapy for HER2-overexpressing metastatic breast cancer: multicenter phase II trial with clinical outcomes, analysis of serum tumor markers as predictive factors, and cardiac surveillance algorithm. J Clin Oncol. 2003;21(15):2889-2895.

21. Bartsch R, Wenzel C, Altorjai G, et al. Capecitabine and trastuzumab in heavily pretreated metastatic breast cancer. J Clin Oncol. 2007;25(25):3853-3858.

22. Schaller G, Fuchs I, Gonsch T, et al. Phase II study of capecitabine plus trastuzumab in human epidermal growth factor receptor 2 overexpressing metastatic breast cancer pretreated with anthracyclines or taxanes. J Clin Oncol. 2007;25(22):3246-3250.

23. Yamamoto D, Iwase S, Kitamura K, Odagiri H, Yamamoto C, Nagumo Y A phase II study of trastuzumab and capecitabine for patients with HER2-overexpressing metastatic breast cancer: Japan Breast Cancer Research Network (JBCRN) 00 Trial. Cancer Chemother Pharmacol. 2008;61(3):509-514

24. Pegram MD, Lipton A, Hayes DF, et al. Phase II study of receptorenhanced chemosensitivity using recombinant humanized anti-p185HER2/ neu monoclonal antibody plus cisplatin in patients with HER2/ neu-overexpressing metastatic breast cancer refractory to chemotherapy treatment. J Clin Oncol. 1998;16(8):2659-2671.

25. Pegram MD, Pienkowski T, Northfelt DW, et al. Results of two openlabel, multicenter phase II studies of docetaxel, platinum salts, and trastuzumab in HER2-positive advanced breast cancer. J Natl Cancer Inst. 2004;96(10):759-769.
26. O’Shaughnessy JA, Vukelja S, Marsland T, Kimmel G, Ratnam S, Pippen JE. Phase II study of trastuzumab plus gemcitabine in chemotherapy-pretreated patients with metastatic breast cancer. Clin Breast Cancer. 2004;5(2):142-147.

27. Von Minckwitz G, Zielinski C, Maarteense E, et al. Capecitabine vs capecitabine + trastuzumab in patients with HER2-positive metastatic breast cancer progressing during trastuzumab treatment: The TBP phase III study (GBG 26/BIG 3-05). J Clin Oncol. 2008; 26(May 20 Suppl):abstr 1025.

28. Piccart-Gebhart MJ, Procter M, Leyland-Jones B, et al. Trastuzumab after adjuvant chemotherapy in HER2-positive breast cancer. $N$ Engl J Med. 2005;353(16):1659-1672.

29. Romond EH, Perez EA, Bryant J, et al. Trastuzumab plus adjuvant chemotherapy for operable HER2-positive breast cancer. $N$ Engl J Med. 2005;353(16):1673-1684.

30. Slamon D, Eiermann W, Robert N, et al. Phase III randomized trial comparing doxorubicin and cyclophosphamide followed by docetaxel (AC T) with doxorubicin and cyclophosphamide followed by docetaxel and trastuzumab $(\mathrm{ACTH})$ with docetaxel, carboplatin and trastuzumab $(\mathrm{TCH})$ in HER2/neu positive early breast cancer patients: BCIRG 006 Study. San Antonio Breast Cancer Symposium. 2005: abstr 1.

31. Joensuu H, Kellokumpu-Lehtinen PL, Bono P, et al. Adjuvant docetaxel or vinorelbine with or without trastuzumab for breast cancer. $N$ Engl J Med. 2006;354(8):809-820.

32. Spielmann M, Roché H, Humblet Y, et al. 3-year follow-up of trastuzumab following adjuvant chemotherapy in node positive HER2positive breast cancer patients: results of the PACS-04 trial. San Antonio Breast Cancer Symposium. 2007(abstr 72).

33. Smith I, Procter M, Gelber RD, et al. 2-year follow-up of trastuzumab after adjuvant chemotherapy in HER2-positive breast cancer: a randomised controlled trial. Lancet. 007;369(9555):29-36.

34. Slamon D, Eiermann W, Robert N, et al. BCIRG 006: 2nd interim analysis phase III randomized trial comparing doxorubicin and cyclophosphamide followed by docetaxel (ACT) with doxorubicin and cyclophosphamide followed by docetaxel and trastuzumab (ACTH) with docetaxel, carboplatin and trastuzumab (TCH) in Her2neu positive early breast cancer patients. Breast Cancer Res Treat. 2006; 106(Suppl 1):52a.

35. Six vs 12 months of transtuzumab with docetaxel following FEC as adjuvant treatment in $\mathrm{N}+$ breast cancer. Accessed online 23 January 2009. http://clinicaltrials.gov/ct2/show/NCT00615602.

36. Trastuzumab for 6 months or 1 year in treating women with nonmetastatic breast cancer that can be removed by surgery. Accessed online 27 th April 2009. http://www.clinicaltrials.gov/ct2/show/NCT00381901.

37. Telli ML, Hunt SA, Carlson RW, Guardino AE. Trastuzumab-related cardiotoxicity: calling into question the concept of reversibility. J Clin Oncol. 2007;25(23):3525-3533.

38. Tan-Chiu E, Yothers G, Romond E, et al. Assessment of cardiac dysfunction in a randomized trial comparing doxorubicin and cyclophosphamide followed by paclitaxel, with or without trastuzumab as adjuvant therapy in node-positive, human epidermal growth factor receptor 2-overexpressing breast cancer: NSABP B-31. J Clin Oncol. 2005;23(31):7811-7819.

39. Ewer MS, Vooletich MT, Durand JB, et al. Reversibility of trastuzumabrelated cardiotoxicity: new insights based on clinical course and response to medical treatment. J Clin Oncol. 2005;23(31):7820-7826.

40. Chen B, Peng X, Pentassuglia L, Lim CC, Sawyer DB. Molecular and cellular mechanisms of anthracycline cardiotoxicity. Cardiovasc Toxicol. 2007;7(2):114-121

41. Trastuzumab FDA label (version approved on 01/18/2008). Accessed online 04/27/2009. Available at http://www.fda.gov/cder/foi/label/ 2008/103792s5175lbl.pdf. 2008.

42. Nahta R, Esteva FJ. HER2 therapy: molecular mechanisms of trastuzumab resistance. Breast Cancer Res. 2006;8(6):215.

43. Scaltriti M, Rojo F, Ocana A, et al. Expression of p95HER2, a truncated form of the HER2 receptor, and response to anti-HER2 therapies in breast cancer. J Natl Cancer Inst. 2007;99(8):628-638. 
44. Ritter CA, Perez-Torres M, Rinehart C, et al. Human breast cancer cells selected for resistance to trastuzumab in vivo overexpress epidermal growth factor receptor and ErbB ligands and remain dependent on the ErbB receptor network. Clin Cancer Res. 2007;13(16):4909-4919.

45. Nagata Y, Lan KH, Zhou X, et al. PTEN activation contributes to tumor inhibition by trastuzumab, and loss of PTEN predicts trastuzumab resistance in patients. Cancer Cell. 2004;6(2):117-127.

46. Clark AS, West K, Streicher S, Dennis PA. Constitutive and inducible Akt activity promotes resistance to chemotherapy, trastuzumab, or tamoxifen in breast cancer cells. Mol Cancer Ther. 2002;1(9):707-717.

47. Nahta R, Takahashi T, Ueno NT, Hung MC, Esteva FJ. P27(kip1) down-regulation is associated with trastuzumab resistance in breast cancer cells. Cancer Res. 2004;64(11):3981-3986.

48. Vazquez-Martin A, Colomer R, Brunet J, Menendez JA. Pharmacological blockade of fatty acid synthase (FASN) reverses acquired autoresistance to trastuzumab (Herceptin by transcriptionally inhibiting 'HER2 super-expression' occurring in high-dose trastuzumab-conditioned SKBR3/Tzb100 breast cancer cells. Int J Oncol. 2007;31(4):769-776.

49. Rusnak DW, Lackey K, Affleck K, et al. The effects of the novel, reversible epidermal growth factor receptor/ErbB-2 tyrosine kinase inhibitor, GW2016, on the growth of human normal and tumor-derived cell lines in vitro and in vivo. Mol Cancer Ther. 2001;1(2):85-94.

50. Xia W, Liu LH, Ho P, Spector NL. Truncated ErbB2 receptor (p95ErbB2) is regulated by heregulin through heterodimer formation with ErbB3 yet remains sensitive to the dual EGFR/ErbB2 kinase inhibitor GW572016. Oncogene. 2004;23(3):646-653.

51. Xia W, Gerard CM, Liu L, Baudson NM, Ory TL, Spector NL. Combining lapatinib (GW572016), a small molecule inhibitor of ErbB1 and ErbB2 tyrosine kinases, with therapeutic anti-ErbB2 antibodies enhances apoptosis of ErbB2-overexpressing breast cancer cells. Oncogene. 2005;24(41):6213-6221.

52. Konecny GE, Pegram MD, Venkatesan N, et al. Activity of the dual kinase inhibitor lapatinib (GW572016) against HER2-overexpressing and trastuzumab-treated breast cancer cells. Cancer Res. 2006;66(3):1630-1639.

53. Xia W, Mullin RJ, Keith BR, et al. Anti-tumor activity of GW572016: a dual tyrosine kinase inhibitor blocks EGF activation of EGFR/erbB2 and downstream Erk1/2 and AKT pathways. Oncogene. 2002;21(41): 6255-6263.

54. Bence AK, Anderson EB, Halepota MA, et al. Phase I pharmacokinetic studies evaluating single and multiple doses of oral GW572016, a dual EGFR-ErbB2 inhibitor, in healthy subjects. Invest New Drugs. 2005;23(1):39-49.

55. Versola M, Burris H, Jones S, et al. Clinical activity of GW572016 in EGF10003 in patients with solid tumors. J Clin Oncol. 2004; 22(14S (July 15 Suppl):3047.

56. Burris H, Taylor C, Jones S, et al. A phase I study of GW572016 in patients with solid tumors. Proc Am Soc Clin Oncol. 2003; 22:abstr 994.

57. Pandite L, Burris H, Jones S, et al. A safety, tolerability, and pharmacokinetic (PK) study of GW572016 in patients with solid tumors. J Clin Oncol. 2004;22(14S (July 15 Suppl):3179.

58. Burris HA, 3rd, Hurwitz HI, Dees EC, et al. Phase I safety, pharmacokinetics, and clinical activity study of lapatinib (GW572016), a reversible dual inhibitor of epidermal growth factor receptor tyrosine kinases, in heavily pretreated patients with metastatic carcinomas. J Clin Oncol. 2005;23(23):5305-5313.

59. Burstein HJ, Storniolo AM, Franco S, et al. A phase II study of lapatinib monotherapy in chemotherapy-refractory HER2-positive and HER2-negative advanced or metastatic breast cancer. Ann Oncol. 2008;19(6):1068-1074.

60. Blackwell KL, Pegram MD, Tan-Chiu E, et al. Single-agent lapatinib for HER2-overexpressing advanced or metastatic breast cancer that progressed on first- or second-line trastuzumab-containing regimens. Ann Oncol. 2009;29:29.

61. Gomez HL, Doval DC, Chavez MA, et al. Efficacy and safety of lapatinib as first-line therapy for ErbB2-amplified locally advanced or metastatic breast cancer. J Clin Oncol. 2008;26(18):2999-3005.
62. Geyer CE, Forster J, Lindquist D, et al. Lapatinib plus capecitabine for HER2-positive advanced breast cancer. $N$ Engl J Med. 2006;355(26): 2733-2743.

63. Cameron D, Casey M, Press M, et al. A phase III randomized comparison of lapatinib plus capecitabine versus capecitabine alone in women with advanced breast cancer that has progressed on trastuzumab: updated efficacy and biomarker analyses. Breast Cancer Res Treat. 2008;112(3):533-543.

64. Di Leo A, Gomez HL, Aziz Z, et al. Phase III, double-blind, randomized study comparing lapatinib plus paclitaxel with placebo plus paclitaxel as first-line treatment for metastatic breast cancer. J Clin Oncol. 2008;26(34):5544-5552.

65. Kurokawa H, Arteaga CL. Inhibition of erbB receptor (HER) tyrosine kinases as a strategy to abrogate antiestrogen resistance in human breast cancer. Clin Cancer Res. 2001;7(12 Suppl):4436s-4442s; discussion $4411 \mathrm{~s}-4412 \mathrm{~s}$.

66. Johnston S, Pegram M, Press M, et al. Lapatinib combined with letrozole vs letrozole alone for front line postmenopausal hormone receptor positive $(\mathrm{HR}+)$ metastatic breast cancer (MBC): first results from the EGF30008 Trial. San Antonio Breast Cancer Symposium. 2008:abstr 46.

67. Mackey J, Kaufman B, Clemens M, et al. Trastuzumab prolongs progressionfree survival in hormone-dependent and HER2-positive metastatic breast cancer. Breast Cancer Res Treat. 2006; 100(Suppl 1):abstr 3.

68. O’Shaughnessy J, Blackwell KL, Burstein H, et al. A randomized study of lapatinib alone or in combination with trastuzumab in heavily pretreated HER2 + metastatic breast cancer progressing on trastuzumab therapy. J Clin Oncol. 2008; 26(May 20 Suppl):abstr 1015.

69. Bendell JC, Domchek SM, Burstein HJ, et al. Central nervous system metastases in women who receive trastuzumab-based therapy for metastatic breast carcinoma. Cancer. 2003;97(12):2972-2977.

70. Gril B, Palmieri D, Bronder JL, et al. Effect of lapatinib on the outgrowth of metastatic breast cancer cells to the brain. J Natl Cancer Inst. 2008;100(15):1092-1103

71. Guarneri V, Frassoldati A, Bruzzi P, et al. Multicentric, randomized phase III trial of two different adjuvant chemotherapy regimens plus three versus twelve months of trastuzumab in patients with HER2positive breast cancer (Short-HER Trial; NCT00629278). Clin Breast Cancer. 2008;8(5):453-456.

72. Tomasello G, de Azambuja E, Dinh P, Snoj N, Piccart-Gebhart M. Jumping higher: is it still possible? The ALTTO trial challenge. Expert Rev Anticancer Ther. 2008;8(12):1883-1890.

73. Dang C, Lin N, Moy B, et al. Dose-dense (DD) doxorubicin and cyclophosphamide (AC) followed by weekly paclitaxel $(\mathrm{P})$ with trastuzumab (T) and lapatinib (L) in HER2/neu-positive breast cancer is not feasible due to excessive diarrhea: updated results. San Antonio Breast Cancer Symposium. 2008:abstr 2108.

74. Schneider C, Sepp-Lorenzino L, Nimmesgern E, et al. Pharmacologic shifting of a balance between protein refolding and degradation mediated by Hsp90. Proc Natl Acad Sci U S A. 1996;93(25):14536-14541.

75. Solit DB, Rosen N. Hsp90: a novel target for cancer therapy. Curr Top Med Chem. 2006;6(11):1205-1214.

76. Maloney A, Workman P. HSP 90 as a new therapeutic target for cancer therapy: the story unfolds. Expert Opin Biol Ther. 2002;2(1):3-24.

77. Basso AD, Solit DB, Munster PN, Rosen N. Ansamycin antibiotics inhibit Akt activation and cyclin D expression in breast cancer cells that overexpress HER2. Oncogene. 2002;21(8):1159-1166.

78. Solit DB, Zheng FF, Drobnjak M, et al. 17-Allylamino-17-demethoxygeldanamycin induces the degradation of androgen receptor and HER-2/neu and inhibits the growth of prostate cancer xenografts. Clin Cancer Res. 2002;8(5):986-993.

79. Banerji U, O’Donnell A, Scurr M, et al. Phase I pharmacokinetic and pharmacodynamic study of 17-allylamino, 17-demethoxygeldanamycin in patients with advanced malignancies. J Clin Oncol. 2005;23(18):4152-4161.

80. Goetz MP, Toft D, Reid J, et al. Phase I trial of 17-allylamino-17demethoxygeldanamycin in patients with advanced cancer. $J$ Clin Oncol. 2005;23(6):1078-1087. 
81. Grem JL, Morrison G, Guo XD, et al. Phase I and pharmacologic study of 17-(allylamino)-17-demethoxygeldanamycin in adult patients with solid tumors. J Clin Oncol. 2005;23(9):1885-1893.

82. Nowakowski GS, McCollum AK, Ames MM, et al. A phase I trial of twice-weekly 17-allylamino-demethoxy-geldanamycin in patients with advanced cancer. Clin Cancer Res. 2006;12(20 Pt 1):6087-6093.

83. Ramanathan RK, Egorin MJ, Eiseman JL, et al. Phase I and pharmacodynamic study of 17-(allylamino)-17-demethoxygeldanamycin in adult patients with refractory advanced cancers. Clin Cancer Res. 2007;13(6):1769-1774.

84. Ramanathan RK, Trump DL, Eiseman JL, et al. Phase I pharmacokineticpharmacodynamic study of 17-(allylamino)-17-demethoxygeldanamycin (17AAG, NSC 330507), a novel inhibitor of heat shock protein 90, in patients with refractory advanced cancers. Clin Cancer Res. 2005;11(9): 3385-3391.

85. Solit DB, Ivy SP, Kopil C, et al. Phase I trial of 17-allylamino-17demethoxygeldanamycin in patients with advanced cancer. Clin Cancer Res. 2007;13(6):1775-1782.

86. Modi S, Stopeck AT, Gordon MS, et al. Combination of trastuzumab and tanespimycin (17-AAG, KOS-953) is safe and active in trastuzumab-refractory HER-2 overexpressing breast cancer: a phase I dose-escalation study. J Clin Oncol. 2007;25(34):5410-5417.

87. Modi S, Sugarman S, Stopeck A, et al. Phase II trial of the Hsp90 inhibitor tanespimycin (Tan) + trastuzumab (T) in patients (pts) with HER2-positive metastatic breast cancer (MBC). J Clin Oncol. 2008; 26(May 20 Suppl):abstr 1027.

88. Franklin MC, Carey KD, Vajdos FF, Leahy DJ, de Vos AM, Sliwkowski MX. Insights into ErbB signaling from the structure of the ErbB2-pertuzumab complex. Cancer Cell. 2004;5(4):317-328.

89. Gelmon KA, Fumoleau P, Verma S, et al. Results of a phase II trial of trastuzumab $(\mathrm{H})$ and pertuzumab $(\mathrm{P})$ in patients (pts) with HER2-positive metastatic breast cancer (MBC) who had progressed during trastuzumab therapy. J Clin Oncol. 2008; 26(May 20 suppl):abstr 1026.
90. Slamon DJ. Proto-oncogenes and human cancers. $N$ Engl J Med. 1987;317(15):955-957.

91. Lewis Phillips GD, Li G, Dugger DL, et al. Targeting HER2-positive breast cancer with trastuzumab-DM1, an antibody-cytotoxic drug conjugate. Cancer Res. 2008;68(22):9280-9290.

92. Beeram M, Krop I, Modi S, et al. A phase I study of trastuzumabMCC-DM1 (T-DM1), a first-in-class HER2 antibody-drug conjugate (ADC), in patients (pts) with HER2 + metastatic breast cancer (BC). J Clin Oncol. 2007;25(18S (June 20 Suppl):abstr 1042.

93. Krop IE, Mita M, Burris HA, et al. A phase I study of weekly dosing of trastuzumab-DM1 (T-DM1) in patients with advanced HER2+ breast cancer. San Antonio Breast Cancer Symposium. 2008(abstr 3136).

94. Vukelja S, Rugo H, Vogel C, et al. A phase II study of trastuzumabDM1, a first-in-class HER2 antibody-drug conjugate, in patients with HER2+ metastatic breast cancer. San Antonio Breast Cancer Symposium. 2008:abstr 33.

95. Burstein HJ, Sun Y, Tan AR, et al. Neratinib (HKI-272), an irreversible pan erbB receptor tyrosine kinase inhibitor: phase 2 results in patients with advanced HER2+ breast cancer. San Antonio Breast Cancer Symposium. 2008:abstr 37.

96. Berns K, Horlings HM, Hennessy BT, et al. A functional genetic approach identifies the PI3K pathway as a major determinant of trastuzumab resistance in breast cancer. Cancer Cell. Oct 2007;12(4):395-402.

97. O'Regan R, Andre F, Campone M, et al. RAD001 (everolimus) in combination with weekly paclitaxel and trastuzumab in patients with HER-2-overexpressing metastatic breast cancer with prior resistance to trastuzumab: a multicenter phase I clinical trial. San Antonio Breast Cancer Symposium. 2008:abstr 3119.

98. Fasolo A, Gianni L, Rorive A, et al. Multicenter phase I clinical trial of daily and weekly RAD001 (everolimus) in combination with vinorelbine and trastuzumab in patients with HER-2-overexpressing metastatic breast cancer with prior resistance to trastuzumab. San Antonio Breast Cancer Symposium. 2008.
Biologics: Targets \& Therapy

\section{Publish your work in this journal}

Biologics: Targets \& Therapy is an international, peer-reviewed journal focusing on the patho-physiological rationale for and clinical application of Biologic agents in the management of autoimmune diseases, cancers or other pathologies where a molecular target can be identified. This journal is indexed on PubMed Central, CAS,

\section{Dovepress}

EMBase, Scopus and the Elsevier Bibliographic databases. The manuscript management system is completely online and includes a very quick and fair peer-review system, which is all easy to use. Visit http://www.dovepress.com/testimonials.php to read real quotes from published authors. 